\title{
A dual characterization of length spaces with application to Dirichlet metric spaces
}

\author{
by \\ Peter Stollmann (Chemnitz)
}

\begin{abstract}
We show that under minimal assumptions, the intrinsic metric induced by a strongly local Dirichlet form induces a length space. The main input is a dual characterization of length spaces in terms of the property that the 1-Lipschitz functions form a sheaf.
\end{abstract}

1. Introduction. The theory of Dirichlet forms originated in the fundamental papers [1, 2] and uses the interplay of probabilistic and analytic techniques. Driven by an ever increasing range of applications it has seen extensions in different directions, as witnessed by the monographs [11, 4, 22, 12]. Since the 90's, the metric geometry of the underlying spaces has been thoroughly investigated (cf. [3, 29, 31]). Let us briefly outline some of the main ideas, referring to the following section for precise definitions. A Dirichlet form $\mathcal{E}$ is, in particular, a closed form on $L^{2}(X)$ and so comes with a selfadjoint operator $H$ in $L^{2}(X)$. The additional properties needed for a Dirichlet form can be rephrased in terms of the semigroup $\left(e^{-t H} ; t \geq 0\right)$ and capture some of the fundamental properties of the heat semigroup that arises in the most important special case $H=-\Delta$. At this point let me mention another favorite of mine, [27, in which the reader can find a lot about Dirichlet forms, and the associated operator semigroup, although Dirichlet forms are not even mentioned. If we start with a local Dirichlet form in a sufficiently regular setting, the form comes with a measure, the energy measure that can be used to define a metric $d_{\mathcal{E}}$ on the space $X$ itself. This metric captures some of the properties of $\mathcal{E}$, the operator $H$ and its semigroup, and generalizes Riemannian distances. The general setup can be used for extensions and a unified treatment of many important aspects of classical analysis: properties of solutions to the abstract analogue of the Laplace equation and the heat equation [3, 29], more recently [7, 13], and also spectral properties

2010 Mathematics Subject Classification: Primary 30Lxx; Secondary 31C25.

Key words and phrases: metric spaces, length metric, Dirichlet forms. 
[6, 5, 19]; here we just picked a few particular references and refer to the literature cited there for a more complete picture.

Here, we contribute to a basic question concerning the metric spaces that arise in this manner, namely the degree of connectedness of the space $\left(X, d_{\mathcal{E}}\right)$. A fundamental result from [31] states that this space is geodesic in the sense that any two points are connected by a path of length $d_{\mathcal{E}}(x, y)$, provided $\left(X, d_{\mathcal{E}}\right)$ is complete. Two questions arise: what happens without this crucial assumption and why should we care? Actually, in several previous papers I have adopted this completeness property without further ado. However, as we will see below, this assumption is not valid for the second most simple class of examples, the classical Dirichlet form on open subsets of Euclidean space $\mathbb{R}^{d}$. Therefore, a treatment of the noncomplete case is more than justified. As we will see in Theorem 5.2, the minimal assumption that $d_{\mathcal{E}}$ is a metric in the wide sense that generates the original topology on $X$ already guarantees that $\left(X, d_{\mathcal{E}}\right)$ is a length space. This means that any two points in this space can be joined by a path with length arbitrarily close to the distance of these points.

In the investigation of the intrinsic metric of Dirichlet forms it turned out that a certain dual object of $X, \operatorname{Lip}^{1}$, the space of 1-Lipschitz functions, plays a central role: more precisely, the question whether Lip $^{1}$ is a sheaf will be crucial for the path metric property of a metric space. Here, we say that $\mathrm{Lip}^{1}$ is a sheaf if every function that is locally 1-Lipschitz is already globally 1-Lipschitz. This leads to an abstract characterization of length spaces that is of independent interest, Theorem 4.2 .

After introducing the necessary notions in the following section, we turn to open subsets of Euclidean space as a class of examples for which we can already illustrate the main questions and ideas. Then we prove the above mentioned dual characterization of length spaces, and the final section is devoted to the proof of the fact that Dirichlet metric spaces are length spaces.

2. Basic notions. A metric $d$ on a set $X$ is a symmetric mapping $d: X \times X \rightarrow[0, \infty)$ such that $d(x, y)=0$ if and only $x=y$, and $d$ satisfies the triangle inequality $d(x, z) \leq d(x, y)+d(y, z)$. If $d$ is allowed to take values in $[0, \infty]$ we speak of a metric in the wide sense. We write

$$
B(x, r):=\{y \in X \mid d(x, y) \leq r\} \quad \text { and } \quad U(x, r):=\{y \in X \mid d(x, y)<r\}
$$

for the closed and open balls, respectively. A continuous mapping $\gamma: I \rightarrow X$ from an interval $I \subset \mathbb{R}$ to $X$ is called a path. A metric in the wide sense induces a length structure in terms of

$$
L(\gamma):=\sup \left\{\sum_{k=0}^{N} d\left(\gamma\left(t_{k+1}\right), \gamma\left(t_{k}\right)\right) \mid t_{0}<t_{1}<\cdots<t_{n}, t_{0}, \ldots, t_{n} \in I\right\} .
$$


The path metric induced by $d$ is given by

$$
d_{\ell}(x, y):=\inf \{L(\gamma) \mid \gamma: I \rightarrow X \text { a path } x, y \in \gamma(I)\} \in[0, \infty],
$$

with the usual convention that $\inf \emptyset=\infty$. See [15] for an axiomatic treatment of length structures that are not based on an a priori given metric. The triangle inequality gives that $d(x, y) \leq d_{\ell}(x, y)$, and we say that $(X, d)$ is a length space provided that $d=d_{\ell}$.

A canonical dual object for a metric space is the space of 1-Lipschitz functions. For $U \subset X$ denote

$$
\operatorname{Lip}^{1}(U, d):=\operatorname{Lip}^{1}(U):=\{f: X \rightarrow \mathbb{R}|| f(x)-f(y) \mid \leq d(x, y) \text { for all } x, y \in U\},
$$
$\operatorname{Lip}_{\text {loc }}^{1}(U, d):=\operatorname{Lip}_{\text {loc }}^{1}(U):=\{f: X \rightarrow \mathbb{R} \mid \forall x \in X \exists V$ open with $x \in V$

$$
\text { such that } \left.\left.f\right|_{V \cap U} \in \operatorname{Lip}^{1}(V \cap U)\right\} \text {. }
$$

Note that $\operatorname{Lip}^{1}(U) \subset \operatorname{Lip}_{\text {loc }}^{1}(U)$ but they are different in general, as can be easily seen by considering a disconnected set $U$. We say that $\mathrm{Lip}^{1}$ is a sheaf on $U$ if $\operatorname{Lip}^{1}(U)=\operatorname{Lip}_{\text {loc }}^{1}(U)$. If that holds for $X=U$ we say that $\operatorname{Lip}^{1}$ is a sheaf. Our first main result, Theorem 4.2 , says that a locally complete space is a length space if and only if $\operatorname{Lip}^{1}$ is a sheaf. It is clear that $d$ can be written as

$$
d(x, y)=\sup \left\{f(y)-f(x) \mid f \in \operatorname{Lip}^{1}(V)\right\}
$$

for every subset $V \subset X$ and all $x, y \in V$. The meaning of this observation will become clear immediately when we discuss the intrinsic metric.

Dirichlet metric spaces. The main application of our dual characterization and the starting point for the present paper are metrics induced by a strongly local, regular Dirichlet form, a notion we now briefly introduce. The starting setup is a locally compact metric space $X$ endowed with a regular Borel measure $m$ and a Dirichlet form $\mathcal{E}$ in $L^{2}(X, m)$. We refer to [4, 11, 12, 22, for a thorough treatment of Dirichlet forms, a notion that goes back to [1, 2]. The example one should keep in mind is the classical Dirichlet form

$$
\mathcal{D}(\mathcal{E}):=W_{0}^{1,2}(\Omega), \quad \mathcal{E}[u, v]=\int_{\Omega} \nabla u(x) \cdot \nabla v(x) d x,
$$

where $\Omega$ is an open subset of $\mathbb{R}^{d}$ and $d x$ denotes integration with respect to Lebesgue measure. A Dirichlet form is called regular if its domain is large enough so that $\mathcal{D}(\mathcal{E}) \cap C_{c}(X)$ is dense both in $\left(C_{c}(X),\|\cdot\|_{\infty}\right)$ and in $\left(\mathcal{D}(\mathcal{E}),\|\cdot\|_{\mathcal{E}}\right)$, where the energy norm $\|\cdot\|_{\mathcal{E}}$ is defined by $\|u\|_{\mathcal{E}}^{2}:=\mathcal{E}[u, u]+\|u\|^{2}$ and can be thought of as an analogue of the first order Sobolev norm, which appears for the classical Dirichlet form, as the reader will immediately notice.

If a regular Dirichlet form is strongly local, i.e., if

$$
\mathcal{E}[u, v]=0 \quad \text { for } u \text { constant on } \operatorname{supp} v,
$$


then $\mathcal{E}$ can be represented in a way quite similar to the classical Dirichlet form. Namely, there exists a bilinear mapping $\Gamma$ from $\mathcal{D}(\mathcal{E}) \times \mathcal{D}(\mathcal{E})$ to the set $\mathcal{M}_{R}$ of signed Radon measures on $X$ such that

$$
\mathcal{E}[u, v]=\int_{X} d \Gamma(u, v) .
$$

This so called energy measure or Lagrangian can be defined via

$$
\int_{X} \phi d \Gamma(u, u)=\mathcal{E}[u, \phi u]-\frac{1}{2} \mathcal{E}\left[u^{2}, \phi\right] \quad \text { for } u, \phi \in \mathcal{D}(\mathcal{E}) \cap C_{c}(X)
$$

and is extended to a bilinear mapping by polarization. In [11, 12], the energy measure is denoted by

$$
\Gamma(u, u)=\mu_{\langle u\rangle} .
$$

It can be extended to

$$
\mathcal{D}_{\text {loc }}:=\left\{f \in L_{\text {loc }}^{2}(X) \mid \forall K \subset X \text { compact } \exists u \in \mathcal{D}(\mathcal{E}):\left.u\right|_{K}=\left.f\right|_{K}\right\}
$$

and inherits several important properties of the underlying Dirichlet form. E.g., $\Gamma$ is strongly local as well, meaning that, for open $V \subset X$ and $f \in \mathcal{D}_{\text {loc }}$, i.e.,

$$
\mathbb{1}_{V} d \Gamma(f, f)=0 \quad \text { whenever } f \text { is constant on } V,
$$

$$
\int \phi d \Gamma(f, f)=0 \quad \text { whenever } f \text { is constant on } V \text {, and } \phi \in C_{c}(V) .
$$

Given the energy measure $\Gamma$, we can finally define the intrinsic metric in the following way: Consider

$$
\mathcal{A}^{1}:=\left\{f \in \mathcal{D}_{\text {loc }} \cap C(X) \mid \Gamma(f, f) \leq m\right\},
$$

where the inequality signifies that $d \Gamma(f, f)$ is absolutely continuous with respect to the underlying measure $m$ with Radon-Nikodým derivative bounded by 1 . In analogy with the classical Dirichlet form, $\mathcal{A}^{1}$ can be thought of as those continuous functions for which the gradient is bounded by 1 in norm. We set

$$
d_{\mathcal{E}}(x, y):=\sup \left\{f(y)-f(x) \mid f \in \mathcal{A}^{1}\right\} \in[0, \infty]
$$

and call it the intrinsic metric induced by $\mathcal{E}$ (see [3, 29, 31]); some properties of the set $\mathcal{A}^{1}$ can be found in the appendix of [5]. We will always assume that $\mathcal{E}$ is strictly local, by which we mean that $d_{\mathcal{E}}$ is a metric in the wide sense and induces the original topology on $X$. In particular, this means that $\mathcal{A}^{1}$ separates the points of $X$. Note that $d_{\mathcal{E}}(x, y)=\infty$ occurs naturally if $x$ and $y$ are in different connected components of $X$, as was also discussed in [31].

3. The classical Dirichlet form on open subsets of $\mathbb{R}^{d}$. Throughout this section $\Omega$ denotes an open subset of $\mathbb{R}^{d}$. We will consider the usual Euclidean metric $\rho$ as well as the intrinsic metric $d_{\mathcal{E}}$ induced by the classical 
Dirichlet form defined above. In that case

$$
d \Gamma(f, f)(x)=|\nabla f(x)|^{2} d x \quad \text { for } f \in W_{\mathrm{loc}}^{1,2}(\Omega),
$$

and

$$
\mathcal{A}^{1}=\left\{f \in W_{\text {loc }}^{1,2}(\Omega) \cap C(\Omega)|| \nabla f(x) \mid \leq 1 \text { a.e. }\right\} .
$$

We write $\rho(x, y)$ for the Euclidean metric on $\mathbb{R}^{d}$ to distinguish it from the other metrics introduced so far.

REMARK 3.1. $(\Omega, \rho)$ is complete if and only if $\Omega=\mathbb{R}^{d}$.

Clearly, $\Omega=\mathbb{R}^{d} \backslash\{0\}$ gives an example of a length space that is not geodesic, as open subsets are geodesic with respect to the Euclidean metric if and only if they are convex.

EXAmple 3.2. Let $\Omega=\mathbb{R}^{2} \backslash(\{0\} \times[-1,1])$. Then $(\Omega, \rho)$ is not a length space. It is also easy to see that $\operatorname{Lip}^{1}(\Omega) \varsubsetneqq \operatorname{Lip}_{\text {loc }}^{1}(\Omega)$. Choose, e.g., $f(x, y):=$ $(1-|(x, y)|)_{+}$for $(x, y)$ in the left halfplane and $f(x, y):=0$ on the right halfplane.

Of course, this example does not come as a surprise in view of Theorem 4.2 below. We now relate the intrinsic metric $d_{\mathcal{E}}$ to the Euclidean metric and obtain an explicit formula. We are not aware of any reference for this simple fact:

Proposition 3.3. For any open subset $\Omega$ of $\mathbb{R}^{d}$ we have $d_{\mathcal{E}}=\rho_{\ell}$, the length metric coming from Euclidean distance.

Proof. Pick $x, y \in \Omega$. If $x$ and $y$ lie in different connected components $U$ and $V$ of $\Omega$ then $\rho_{\ell}(x, y)=\infty$, since there is no path joining the two points. But $d_{\mathcal{E}}(x, y)=\infty$ as well, as can be seen from picking $f_{n}:=n \mathbb{1}_{V} \in \mathcal{A}^{1}$ as trial functions:

$$
d_{\mathcal{E}}(x, y) \geq \sup _{n \in \mathbb{N}}\left(f_{n}(y)-f_{n}(x)\right)=\infty .
$$

Suppose now $\rho_{\ell}(x, y)<\infty$. Since $\Omega$ is open, we can find $r>0$ such that $B(x, r) \subset \Omega$. Since the ball is convex we see that

$$
\left|\rho_{\ell}\left(x, y_{0}\right)-\rho_{\ell}\left(x, y_{1}\right)\right| \leq\left|y_{0}-y_{1}\right| \quad \text { for } y_{0}, y_{1} \in B(x, r),
$$

which implies that $f: \Omega \rightarrow \mathbb{R}, f(y):=\rho_{\ell}(x, y)$ for $\rho_{\ell}(x, y)<\infty$ and 0 elsewhere, defines a function in $\mathcal{A}^{1}$. This gives

$$
d_{\mathcal{E}}(x, y) \geq f(y)-f(x)=\rho_{\ell}(x, y) .
$$

Conversely, let $f \in \mathcal{A}^{1}$ and $\gamma:[0,1] \rightarrow \Omega$ be a polygonal path from $x$ to $y$. 
Then

$$
\begin{aligned}
f(y)-f(x) & =f(\gamma(1))-f(\gamma(0))=\int_{0}^{1} f^{\prime}(\gamma(t)) \gamma^{\prime}(t) d t \\
& \leq \int_{0}^{1}\left|\gamma^{\prime}(t)\right| d t=L(\gamma) .
\end{aligned}
$$

By taking the inf over all paths $\gamma$, we see that

$$
f(y)-f(x) \leq \rho_{\ell}(x, y) .
$$

This gives $d_{\mathcal{E}}(x, y) \leq \rho_{\ell}(x, y)$.

Closely related is, e.g., Lemma I.1.24 from [28] where divergence forms are treated, in the case $X=\mathbb{R}^{d}$, however. Since we have now calculated $d_{\mathcal{E}}$ explicitly, we can record some simple consequences:

REMARK 3.4. Like for the Euclidean distance, $\left(\Omega, d_{\mathcal{E}}\right)$ is complete if and only if $\Omega=\mathbb{R}^{d}$. For arbitrary $\Omega,\left(\Omega, d_{\mathcal{E}}\right)$ is a length space, since $\left(\rho_{\ell}\right)_{\ell}=\rho_{\ell}$ for any metric; see [15, 1.6].

This gives a family of natural examples for which Dirichlet metrics are locally complete but not complete. In particular, our Theorem 5.2 below, stating that all Dirichlet metrics define length spaces, cannot be obtained from any Hopf-Rinow type result, as was done in [31] under the additional assumption of completeness. See also [15, 25].

4. Path metric property and $\mathrm{Lip}^{1}$. In order to find a path with length close to the distance between $x$ and $y$ in a metric space one has to manage to find approximate midpoints, i.e., for every $x$ and $y$ and $\varepsilon>0$ there should be a $z$ such that

$$
d(x, z) \leq \frac{1}{2} d(x, y)+\varepsilon \quad \text { and } \quad d(z, y) \leq \frac{1}{2} d(x, y)+\varepsilon .
$$

We will now see that this property follows from the sheaf property of Lip ${ }^{1}$. In this section, $(X, d)$ denotes a metric space.

Proposition 4.1. Let $U$ be an open subset of $X$ such that $\operatorname{Lip}^{1}(U)=$ $\operatorname{Lip}_{\text {loc }}^{1}(U)$. Then $B\left(x, r_{1}\right) \cap B\left(y, r_{2}\right) \neq \emptyset$ for $d(x, y)<r_{1}+r_{2}$. In particular, we can find approximate midpoints in $U$.

Proof. Assume that $B\left(x, r_{1}\right) \cap B\left(y, r_{2}\right)=\emptyset$. Let $\delta>0$ be such that $B\left(x, r_{1}-\delta\right) \cap B\left(y, r_{2}-\delta\right)=\emptyset$. Consider the function

$$
f(z):=-\left(\left(r_{1}-\delta\right)-d(x, z)\right)_{+}+\left(\left(r_{2}-\delta\right)-d(z, y)\right)_{+} \cdot
$$

It is clear that $f(y)=r_{2}-\delta, f(x)=-\left(r_{1}-\delta\right)$. Moreover, $f \equiv 0$ on $W:=U \backslash\left(B\left(x, r_{1}-\delta\right) \cup B\left(y, r_{2}-\delta\right)\right)$. Using the covering $U\left(x, r_{1}\right), U\left(y, r_{2}\right), W$ we see that $f \in \operatorname{Lip}_{\text {loc }}^{1}(U)$. Since $\operatorname{Lip}^{1}(U)=\operatorname{Lip}_{\text {loc }}^{1}(U)$, we find that $d(x, y) \geq$ 
$f(y)-f(x)=r_{1}+r_{2}-2 \delta$. As $\delta$ was arbitrary, this proves that $d(x, y) \geq$ $r_{1}+r_{2}$, as we wanted to show.

Although the proof is clearly inspired by the proof of Lemma 3 in [31, both the assumption and the assertion are in fact quite different. It could also and will be used for Dirichlet metric spaces, where the defining function class $\mathcal{A}^{1}$ is a sheaf. Here is our first main result:

Theorem 4.2. If $(X, d)$ is a length space then $\operatorname{Lip}^{1}$ is a sheaf. Conversely, if $(X, d)$ is locally complete and $\operatorname{Lip}^{1}$ is a sheaf then $(X, d)$ is a length space.

Proof. Assume that $(X, d)$ is a length space and let $f \in \operatorname{Lip}_{\text {loc }}^{1}$. Let $x, y \in X$ and $\varepsilon>0$. Then there is a path $\gamma:[0,1] \rightarrow X$ such that $\gamma(0)=x$ and $\gamma(1)=y$ and $L(\gamma) \leq d(x, y)+\varepsilon$. For every $t \in[0,1]$ there is an open neighborhood $U_{\gamma(t)}$ of $\gamma(t)$ such that $\left.f\right|_{U_{\gamma(t)}} \in \operatorname{Lip}^{1}\left(U_{\gamma(t)}\right)$, since $f \in \operatorname{Lip}_{\text {loc }}^{1}$. By continuity of $\gamma$, there is an open (in $[0,1]$ ) interval $I_{t}$ containing $t$ such that $\gamma\left(I_{t}\right) \subset U_{\gamma(t)}$. Compactness of $[0,1]$ yields finitely many $0=t_{1}<\cdots<$ $t_{m}=1$ such that

$$
[0,1] \subset I_{t_{1}} \cup \cdots \cup I_{t_{m}},
$$

and we may assume that this covering is minimal. Observe that consequently $I_{t_{j}} \cap I_{t_{j+1}} \neq \emptyset$ for $j=1, \ldots, m-1$.

Pick $s_{1}, \ldots, s_{m-1} \in[0,1]$ such that

$$
\begin{aligned}
& 0 \leq t_{1}<s_{1}<t_{2}<\cdots<s_{m-1}<t_{m}=1 \\
& \quad \text { with } s_{j} \in I_{t_{j}} \cap I_{t_{j+1}} \text { for } j=1, \ldots, m-1 .
\end{aligned}
$$

Then

$$
\gamma\left(s_{j}\right) \in U_{\gamma\left(t_{j}\right)} \cap U_{\gamma\left(t_{j+1}\right)} .
$$

Using the triangle inequality and the 1-Lipschitz property in the appropriate neighborhoods, we get

$$
\begin{aligned}
|f(x)-f(y)| & \leq \sum_{j=1}^{m-1}\left(\left|f\left(\gamma\left(t_{j}\right)\right)-f\left(\gamma\left(s_{j}\right)\right)\right|+\left|f\left(\gamma\left(s_{j}\right)\right)-f\left(\gamma\left(t_{j+1}\right)\right)\right|\right) \\
& \leq \sum_{j=1}^{m-1}\left(d\left(\gamma\left(t_{j}\right), \gamma\left(s_{j}\right)\right)+d\left(\gamma\left(s_{j}\right), \gamma\left(t_{j+1}\right)\right)\right) \\
& \leq L(\gamma) \leq d(x, y)+\varepsilon .
\end{aligned}
$$

Since $\varepsilon>0$ was arbitrary, $|f(x)-f(y)| \leq d(x, y)$.

To prove the converse, we want to show that, for fixed $x \in X$, the function

$$
f: X \rightarrow \mathbb{R}, \quad f(y)=d_{\ell}(x, y)
$$


is finite everywhere (!!) and belongs to Lip 1 loc. (Note that, at the moment, it is not even clear that $X$ is pathwise connected.) If this is accomplished, we deduce that $f \in \operatorname{Lip}^{1}$ using our assumption that $\operatorname{Lip}^{1}$ is a sheaf, and $f \in \operatorname{Lip}^{1}$ gives the desired inequality:

$$
d(x, y) \geq|f(y)-f(x)|=d_{\ell}(x, y) .
$$

The first thing we check is the following:

Claim. For every $y \in X$ there exists $r>0$ such that for all $y_{0}, y_{1} \in$ $B(y, r)$,

$$
\left|d_{\ell}\left(x, y_{0}\right)-d_{\ell}\left(x, y_{1}\right)\right| \leq d\left(y_{0}, y_{1}\right) .
$$

We have to find $r>0$ such that any two points $y_{0}, y_{1} \in B(y, r)$ can be joined by a path with length arbitrarily close to $d\left(y_{0}, y_{1}\right)$, since this obviously implies the claim. The existence of approximate midpoints settled in the preceding proposition plus completeness (which we do have locally) will allow us to construct the desired path. This argument is well established: see [15, Theorem 1.8]. Here are the details:

Take $r>0$ so small that $B(y, 3 r)$ is complete; let $y_{0}, y_{1} \in B(y, r)$ and $\delta:=d\left(y_{0}, y_{1}\right)$. Fix $0<\varepsilon<\delta$. Denote

$$
\begin{aligned}
D_{n} & :=\left\{k 2^{-n} \mid k=0, \ldots, 2^{n}\right\} \quad \text { for } n \in \mathbb{N}_{0}, \\
D & :=\bigcup_{n \in \mathbb{N}_{0}} D_{n}=\{\text { dyadic rationals in }[0,1]\} .
\end{aligned}
$$

By induction on $n$ we prove

Assertion $\mathrm{A}(n)$. For all $s \in D_{n}$ there is $y_{s} \in B(y, 3 r)$ such that

$$
d\left(y_{k 2^{-n}}, y_{(k+1) 2^{-n}}\right) \leq 2^{-n}\left(\delta+\varepsilon \sum_{k=1}^{n} 2^{-k}\right) .
$$

For $n=0$, the given points $y_{0}, y_{1}$ satisfy the inequality (the empty sum is interpreted as 0 as usual).

Assume that $\mathrm{A}(n)$ holds and take $s \in D_{n+1}, s=k 2^{-(n+1)}$. For $k$ even, $s \in D_{n}$, so that $y_{s}$ is already defined. Therefore assume that $k$ is odd, so that $y_{(k-1) 2^{-(n+1)}}$ and $y_{(k+1) 2^{-(n+1)}}$ are defined, with distance

$$
d\left(y_{(k-1) 2^{-(n+1)}}, y_{(k+1) 2^{-(n+1)}}\right) \leq 2^{-n}\left(\delta+\varepsilon \sum_{k=1}^{n} 2^{-k}\right),
$$

by what $\mathrm{A}(n)$ gives us. Choose

$$
r_{1}=r_{2}=\frac{1}{2} 2^{-n}\left(\delta+\varepsilon \sum_{k=1}^{n} 2^{-k}\right)+\varepsilon 4^{-(n+1)}
$$


so that $r_{1}+r_{2}>d\left(y_{(k-1) 2^{-(n+1)}}, y_{(k+1) 2^{-(n+1)}}\right)$. From Proposition 4.1 we get a point $y_{k 2^{-(n+1)}} \in B\left(y_{(k-1) 2^{-(n+1)}}, r_{1}\right) \cap B\left(y_{(k+1) 2^{-(n+1)}}, r_{2}\right)$; in particular,

$$
d\left(y_{(k-1) 2^{-(n+1)}}, y_{k 2^{-(n+1)}}\right) \leq r_{1}=2^{-(n+1)}\left(\delta+\varepsilon \sum_{k=1}^{n+1} 2^{-k}\right),
$$

and an analogous bound on $d\left(y_{k 2^{-(n+1)}}, y_{(k+1) 2^{-(n+1)}}\right)$, so that $\mathrm{A}(n+1)$ is proven, since $y_{s} \in B(y, 3 r)$ follows from $(\star)$. Now

$$
\gamma_{0}: D \rightarrow B(y, 3 r), \quad s \mapsto y_{s},
$$

is easily seen to be Lipschitz continuous with Lipschitz constant $\delta+\varepsilon$; note that $(\star)$ gives the coresponding estimate for successive points in $D_{n}$. Since $D$ is dense in $[0,1]$ and $B(y, 3 r)$ is complete, $\gamma_{0}$ extends to a Lipschitz continuous $\gamma:[0,1] \rightarrow B(y, 3 r)$ with Lipschitz constant $\delta+\varepsilon$ and $\gamma(0)=y_{0}$, $\gamma(1)=y_{1}$. The length (as for all Lipschitz continuous paths) can easily be estimated by $\delta+\varepsilon$, settling the claim.

We now show that $f$ is properly defined, that is, $d_{\ell}(x, y)<\infty$ for all $y \in X$; denote $X_{0}:=\left\{y \in X \mid d_{\ell}(x, y)<\infty\right\}$.

As we already observed above, $X$ is connected since $\operatorname{Lip}^{1}$ is a sheaf. From the claim we infer that $X_{0}$ is open and closed. Since $x \in X_{0}, X_{0}$ is nonempty and so must coincide with $X$.

5. Strictly local Dirichlet spaces are length spaces. We now consider the setup introduced in Section 1 above: $X$ is a locally compact space, $\mathcal{E}$ a strictly local Dirichlet form and so comes with an energy measure $\Gamma$ for which

$$
\mathcal{A}^{1}=\left\{f \in \mathcal{D}_{\text {loc }} \cap C(X) \mid \Gamma(f, f) \leq m\right\}
$$

separates the points of $X$ and

$$
d(x, y)=d_{\mathcal{E}}(x, y)=\sup \left\{f(y)-f(x) \mid f \in \mathcal{A}^{1}\right\} \in[0, \infty]
$$

defines a metric in the wide sense that induces the original topology on $X$. In particular, under these assumptions, small enough balls will be compact and hence complete. Clearly,

$$
\mathcal{A}^{1} \subset \operatorname{Lip}^{1} .
$$

The converse is also true, as was established in [10]. Since this work is not yet available, we give a proof in the appendix:

Theorem 5.1 (Rademacher's Theorem). If $d$ is the intrinsic metric of a strictly local Dirichlet form $\mathcal{E}$ on $X$, then

$$
\operatorname{Lip}^{1}=\operatorname{Lip}_{\text {loc }}^{1}=\mathcal{A}^{1} .
$$


The name is due to Rademacher's work [24, who established, for Euclidean space, that a Lipschitz continuous function has bounded weak derivatives. See the lecture notes [16] for more references and [26] for a special case, the configuration space.

Theorem 5.2. If $d$ is the intrinsic metric of a strictly local Dirichlet form $\mathcal{E}$ on $X$, then $(X, d)$ is a length space.

Proof. The only difficulty we have to overcome is that both $d$ and the corresponding path metric $d_{\ell}$ may take the value $\infty$. But, as we will see, that happens simultaneously. We fix $x \in X$.

STEP 1. d admits approximate midpoints.

This follows from Proposition 4.1, keeping in mind that the property defining $\mathcal{A}^{1}$ is local and so $\mathcal{A}^{1}$ is a sheaf (see the Lemma in the Appendix).

SteP 2. For every $y \in X$ there is $r>0$ such that

$$
d\left(y_{0}, y_{1}\right)=d_{\ell}\left(y_{0}, y_{1}\right) \quad \text { for all } y_{0}, y_{1} \in B(y, r) .
$$

This follows from local completeness and the existence of approximate endpoints exactly as in the proof of Theorem 4.2. Again as in that proof we get

STEP 3. The set $Y:=\left\{y \in X \mid d_{\ell}(x, y)<\infty\right\}$ is open and closed.

STEP 4. If $d(x, y)<\infty$ then $d_{\ell}(x, y)<\infty$ as well.

In fact, if $d_{\ell}(x, y)=\infty$, we have $y \notin Y$ and $x \in Y$. Since $Y$ is open and closed, it follows that $n \mathbb{1}_{Y} \in \mathcal{A}^{1}$ (since $d \Gamma\left(\mathbb{1}_{Y}, \mathbb{1}_{Y}\right)=0$ by locality) for all $n \in \mathbb{N}$. Therefore,

$$
d(x, y) \geq n \mathbb{1}_{Y}(x)-n \mathbb{1}_{Y}(y)=n \quad \text { for all } n \in \mathbb{N} .
$$

SteP 5. Let $f: X \rightarrow \mathbb{R}, f(y):=d_{\ell}(x, y)$ for $y \in Y$ and 0 elsewhere. Then $f \in \mathcal{A}^{1}$.

By what we saw in the second step, $f \in \operatorname{Lip}_{\text {loc }}^{1}$. Since $\operatorname{Lip}_{\text {loc }}^{1} \subset \mathcal{A}^{1}$ as mentioned above, we get the desired property of $f$.

We can now finish the proof as follows: The estimate $d(x, y) \leq d_{\ell}(x, y)$ is clear. If $d_{\ell}(x, y)=\infty$, we know from Step 4 that $d(x, y)=\infty$ as well. Thus it remains to consider $d(x, y)<\infty$, in other words $y \in Y$. But this gives

$$
d(x, y) \geq f(y)-f(x)=d_{\ell}(x, y)
$$

for $f$ as above.

REMARK 5.3. In Step 1 of the preceding proof we could also have used a local version of Sturm's Lemma 3 from [31. Conversely, the property proved in the first step combined with compactness easily gives midpoints and this implies that one gets local minimizing geodesics. 
Apart from the examples coming from the classical Dirichlet form, there are many further classes of examples that fall into the framework covered by the preceding result. We mention second order elliptic operators [3, 22, 30, subelliptic operators [8, 9, 17, 18, 23 and Laplace-Beltrami operators on manifolds.

The above results were obtained in connection with a joint work on connectedness and irreducibility properties of Dirichlet forms with D. Lenz and I. Veselić [21].

Appendix. Let us first record some consequences of strict locality; see [19, 20] for background material. Denote, for open $U \subset X$,

$$
\mathcal{A}^{1}(U):=\left\{v \in \mathcal{D}_{\text {loc }}(U) \cap C(U) \mid \Gamma(u, u) \leq m\right\} .
$$

LEMMA 5.4.

(1) $\mathcal{A}^{1}$ is a sheaf, i.e., $f \in \mathcal{A}^{1}$ if and only if for all $x \in X$ there is an open neighborhood $U$ such that $\left.f\right|_{U} \in \mathcal{A}^{1}(U)$.

(2) If $\left(u_{n}\right)_{n \in \mathbb{N}}$ is a sequence in $\mathcal{A}^{1}(U)$ that converges uniformly to $u$, then $u \in \mathcal{A}^{1}(U)$.

Proof. Since the continuous functions are a sheaf and $\Gamma$ is local, (1) is evident.

For the proof of (2), we have to show that $u \in \mathcal{D}_{\text {loc }}(U)$ and $\Gamma(u, u) \leq m$. Both assertions are local. So let $K \subset U$ be compact. Take a compact neighborhood $V \subset U$ of $K$ and use the regularity of $\mathcal{E}$ to find $\eta \in \mathcal{D}(\mathcal{E}) \cap C_{c}(U)$ that is 1 on $V$. Locality of $\Gamma$ gives $\Gamma\left(u_{n} \eta, u_{n} \eta\right) \leq m$ on the interior of $V$. The Leibniz rule guarantees that $\mathcal{E}\left(u_{n} \eta, u_{n} \eta\right)$ remains bounded in $n \in \mathbb{N}$. As the balls in $\left(\mathcal{D}(\mathcal{E}),\|\cdot\|_{\mathcal{E}}\right)$ are weakly compact, there is a subsequence of $\left(u_{n} \eta\right)_{n \in \mathbb{N}}$ converging weakly to some $v \in \mathcal{D}(\mathcal{E})$. Uniform convergence of the $u_{n}$ gives $v=u \eta$, so that there is $v \in \mathcal{D}(\mathcal{E})$ that agrees with $u$ on $K$. The estimate $\Gamma\left(u_{n} \eta, u_{n} \eta\right) \leq m$ on the interior of $V$ carries over to the limit, so that $\Gamma(u, u) \leq m$ on the interior of $V$. As $K$ was arbitrary, that settles the assertion.

The following proof is essentially taken from [10, where a more general result is proven in the sense that certain non-local forms are allowed.

Proof of Theorem 5.1. Fix $f \in \operatorname{Lip}_{\text {loc }}^{1}$. We use the preceding lemma. So it suffices to find, for every point $x$, an open neighborhood $U$ with $\left.f\right|_{U} \in$ $\mathcal{A}^{1}(U)$. Pick $U$ so that $f \in \operatorname{Lip}^{1}(U)$, which is possible by the local Lipschitz property. Furthermore, we can assume that $U$ is relatively compact, since $X$ is locally compact. It follows that $f$ is bounded on $U$, say $\sup _{U}|f| \leq M \in \mathbb{N}$. We now define an approximating sequence for $u:=\left.f\right|_{U}$ in the following way: for $n \in \mathbb{N}$ and $m=-M n, \ldots, M n$ denote $F_{n, m}:=\{y \in X \mid f(y) \geq m / n\}$ and $u_{n, m}:=\left(m / n-d\left(\cdot, F_{n, m}\right)\right)_{+}$, restricted to $U$. The distance $d(\cdot, F)$ is in 
$\mathcal{A}^{1}$ for every closed set (see the appendix of [5]). Therefore, $u_{n, m}$ is in $\mathcal{A}^{1}(U)$ as also is

$$
u_{n}:=\max _{m=-M n, \ldots, M n} u_{n, m} .
$$

Since $f \in \operatorname{Lip}^{1}(U)$ it is easy to see that $u_{n} \rightarrow u$ uniformly. An appeal to part (2) of the preceding lemma finishes the proof.

Acknowledgements. It is a pleasure to thank Ivan Veselić who introduced me to the question that is answered here; thanks are also due to the referee for helpful criticism. Moreover, fruitful discussions with Daniel Lenz, Daniel Wingert and Maria are gratefully acknowledged.

\section{References}

[1] A. Beurling et J. Deny, Espaces de Dirichlet, Acta Math. 99 (1958), 203-224.

[2] - - - Dirichlet spaces, Proc. Nat. Acad. Sci. U.S.A. 45 (1959), 208-215.

[3] M. Biroli and U. Mosco, A Saint-Venant type principle for Dirichlet forms on discontinuous media, Ann. Mat. Pura Appl. (4) 169 (1995), 125-181.

[4] N. Bouleau and F. Hirsch, Dirichlet Forms and Analysis on Wiener Space, de Gruyter, Berlin, 1991.

[5] A. Boutet de Monvel, D. Lenz and P. Stollmann, Schnol's theorem for strongly local forms, Israel J. Math. 173 (2009), 189-211.

[6] A. Boutet de Monvel and P. Stollmann, Eigenfunction expansions for generators of Dirichlet forms, J. Reine Angew. Math. 561 (2003), 131-144.

[7] A. F. M. ter Elst, D. W. Robinson and A. Sikora, Small time asymptotics of diffusion processes, J. Evol. Equations 7 (2007), 79-112.

[8] C. L. Fefferman and D. Phong, Subelliptic eigenvalue problems, in: Conference on Harmonic Analysis in Honor of Antoni Zygmund (Chicago, IL, 1981), W. Beckner et al. (eds.), Wadsworth, 1983, 590-606.

[9] C. L. Fefferman and A. Sánchez-Calle, Fundamental solutions for second order subelliptic operators, Ann. of Math. 124 (1986), 247-272.

[10] R. Frank, D. Lenz and D. Wingert, Intrinsic metrics for (non-local) Dirichlet forms and applications, in preparation.

[11] M. Fukushima, Dirichlet Forms and Markov Processes, North-Holland, Amsterdam, 1980.

[12] M. Fukushima, Y. Oshima, and M. Takeda, Dirichlet Forms and Symmetric Markov Processes, de Gruyter, 1994.

[13] A. Grigor'yan and T. Kumagai, On the dichotomy in the heat kernel two sided estimates, in: Analysis on Graphs and its Applications, Proc. Sympos. Pure Math. 77, Amer. Math. Soc., Providence, RI, 2008, 199-210.

[14] M. Gromov, Structures métriques pour les variétés riemanniennes, Cedic/Nathan, 1981.

[15] —, Metric Structures for Riemannian and Non-Riemannian Spaces, Birkhäuser, Boston, 2007.

[16] J. Heinonen, Lectures on Lipschitz Analysis, Lectures at the 14th Jyväskylä Summer School in August 2004; http://www.math.jyu.fi/research/reports/rep100.pdf

[17] D. Jerison, The Poincaré inequality for vector fields satisfying Hörmander's condition, Duke J. Math. 53 (1986), 503-523. 
[18] D. Jerison and A. Sánchez-Calle, Estimates for the heat kernel for a sum of squares of vector fields, Indiana Univ. Math. J. 35 (1986), 835-854.

[19] D. Lenz, P. Stollmann and I. Veselić, The Allegretto-Piepenbrink theorem for strongly local Dirichlet forms, Doc. Math. 14 (2009), 167-189.

[20] —, - - , Generalized eigenfunctions and spectral theory for strongly local Dirichlet forms, in: OTAMP 2008 proceedings, to appear; arXiv:0909.1107.

[21] - - - - Irreducibility and connectedness for Dirichlet forms, in preparation, 2008.

[22] Z. M. Ma and M. Röckner, Introduction to the Theory of (Nonsymmetric) Dirichlet Forms, Universitext, Springer, Berlin, 1992.

[23] A. Nagel, E. M. Stein and S. Wainger, Balls and metrics defined by vector fields. I: Basic properties, Acta Math. 155 (1985), 103-147.

[24] H. Rademacher, Über partielle und totale Differenzierbarkeit von Funktionen mehrerer Variabeln und über die Transformation der Doppelintegrale, Math. Ann. 79 (1919), 340-359.

[25] W. Rinow, Die innere Geometrie der metrischen Räume, Springer, Berlin, 1961.

[26] M. Röckner and A. Schied, Rademacher's theorem on configuration spaces and applications, J. Funct. Anal. 169 (1999), 325-356.

[27] E. M. Stein, Topics in Harmonic Analysis Related to the Littlewood-Paley Theory, Ann. of Math. Stud. 63, Princeton Univ. Press, Princeton, 1970.

[28] D. W. Stroock, Diffusion semigroups corresponding to uniformly elliptic divergence form operators, in: Séminaire de probabilités, XXII, Lecture Notes in Math. 1321, Springer, 1988, 316-347.

[29] K.-T. Sturm, Analysis on local Dirichlet spaces. I: Recurrence, conservativeness and $L^{p}$-Liouville properties, J. Reine Angew. Math. 456 (1994), 173-196.

[30] - Sharp estimates for capacities and applications to symmetric diffusions, Probab. Theory Related Fields 103 (1995), 73-89.

[31] —, On the geometry defined by Dirichlet forms, in: Seminar on Stochastic Analysis, Random Fields and Applications (Ascona, 1993), E. Bolthausen et al. (eds.), Progr. Probab. 36, Birkhäuser, Boston, 1995, 231-242.

Peter Stollmann

Fakultät für Mathematik

Technische Universität

09107 Chemnitz, Germany

E-mail: peter.stollmann@mathematik.tu-chemnitz.de

Received June 23, 2009

Revised version January 29, 2010 